\title{
The Regional Inequity of $\mathrm{CO}_{2}$ Emissions per Capita in China
}

\author{
Ting Liu ${ }^{1} \&$ Wenqing Pan $^{1}$ \\ ${ }^{1}$ Department of Economics, School of Economics and Management, Tsinghua University, Beijing, China \\ Correspondence: Ting Liu, Department of Economics, School of Economics and Management, Tsinghua \\ University, Beijing 100084, China. Tel: 86-188-1064-4027. E-mail: liut.11@ sem.tsinghua.edu.cn
}

Received: May 8, 2017

doi:10.5539/ijef.v9n7p228
Accepted: May 31, 2017

Online Published: June 25, 2017

URL: https://doi.org/10.5539/ijef.v9n7p228

\begin{abstract}
This paper combines Theil index method with factor decomposition technique to analyze China eight regions' inequality of $\mathrm{CO}_{2}$ emissions per capita, and discuss energy structure, energy intensity, industrial structure, and per capita output's impacts on inequality. This research shows that: (1) The trend of China regional carbon inequality is in the opposite direction to the per capita $\mathrm{CO}_{2}$ emission level. Namely, as the per capita $\mathrm{CO}_{2}$ emission levels rise, regional carbon inequality decreases, and vice versa. (2) Per capita output factor reduces regional carbon inequality, whereas energy structure factor and energy intensity factor increase the inequality. (3) More developed areas can reduce the carbon inequality by improving the energy structure, whereas the divergence of energy intensity in less developed areas has increased to expand the carbon inequity. Thus, when designing $\mathrm{CO}_{2}$ emission reduction targets, policy makers should consider regional differences in economic development level and energy efficiency, and refer to the main influencing factors. At the same time, upgrading industrial structure and upgrading energy technologies should be combined to meet the targets of economic growth and $\mathrm{CO}_{2}$ emission reduction.
\end{abstract}

Keywords: $\mathrm{CO}_{2}$ emissions, energy economy, regional inequality, Theil index, KAYA identity

\section{Introduction}

As the fuel for economic development, fossil energy has been heavily used in the process of production and living, which makes the emission of greenhouse gases such as $\mathrm{CO}_{2}$ in the atmosphere increases, intensifying the greenhouse effect, leading to consequences such as global temperatures rising, threatening both human and civilization survivals. Meanwhile, as one of thorny problems in environmental protection, the rising of $\mathrm{CO}_{2}$ emissions is also closely related to the reduction task allocation, from the perspectives of diverse political and economic interests, which widely attracts the attentions of international organizations and communities, among which China also play an important role. China promised at Copenhagen Conference in 2009 to reduce $\mathrm{CO}_{2}$ per unit of GDP by $40 \%$ to $45 \%$ till 2020 comparing with 2005. At the same time, China in 2016, was the first to sign on the Paris Agreement, making a commitment to control the range of global temperature rising within $2^{\circ} \mathrm{C}$ with more than 170 countries.

In China, different regions share uneven economic developments and energy usage, the regional $\mathrm{CO}_{2}$ emission characteristics without doubt are also different. Therefore, China should consider regional differences when conducting $\mathrm{CO}_{2}$ emission reduction. Facing the daunting task of $\mathrm{CO}_{2}$ reduction, to fairly define regional carbon responsibility, policy makers need to focus on the characteristics of different regions, and to clarify the main driving factors behind carbon emissions together with their trends. Due to different levels of economic development, carbon emissions reveal regional types. The inequality of $\mathrm{CO}_{2}$ emissions among regions reflects regional features, also provides the government with a reference basis to policy making. Combing with the factor decomposition analysis, the regional carbon emission inequality makes the regional characteristics of $\mathrm{CO}_{2}$ emissions richer, and can provide a multi-dimensional reference for policy making. Consequently, considering driving factors behind carbon emissions to study regional inequality offers a fair way to allocate carbon reduction targets, and motivates agents to be more widely and actively involved.

Among various research methods, Theil index (Theil, 1967) has been widely used. Its excellent properties (Bourguignon, 1979), and ability to be combined with decomposition analysis to define each factor's inequity index, make it suiTable for related research topics. Meanwhile, in the studies of regional carbon emission inequality, $\mathrm{CO}_{2}$ per capita is one of indicators accepting growing concerns (Duro \& Padilla, 2006). Factor 
decomposition technique systematically combines the KAYA Identity (1989) with index decomposition methods, can analyze the impacts of carbon intensity, energy structure, energy efficiency, industrial structure and economic growth on $\mathrm{CO}_{2}$ emissions and their changing trends with time. Duro and Padilla (2006) initially combine Theil index and factor decomposition technique, decompose the inequity index of $\mathrm{CO}_{2}$ per capita into KAYA factor inequity index. Also, they illustrate how this method can be applied to empirical studies on inter-regional and intra-regional inequity analysis. Using these methods, this paper will analyze China's inter-regional and intra-regional inequities of per capita carbon emissions.

This paper is organized as follows: section 2 will introduced the methodology and data, in section 3, we will analyze China 8 regions' inter and intra-regional inequality of per capita $\mathrm{CO}_{2}$ and relevant influencing factor inequity index, section 4 makes a conclusion, and corresponding policy Suggestions are given.

\section{Method and Data}

There is no direct observation data of $\mathrm{CO}_{2}$ emissions in China, this paper adapts the method widely used by domestic and foreign scholars to estimate the $\mathrm{CO}_{2}$ emissions in each region of China. For $\mathrm{m}$ sectors and $\mathrm{n}$ types of energy, according to the General Principles of Comprehensive Energy Calculation in China (2008), we firstly calculate the standard coal and calorific value of energy usage. Secondly, according to the IPCC (2006) carbon emission factors, we estimate the total volume of $\mathrm{CO}_{2}$ emissions. Particularly, IPCC (2006) distinguishes between fixed and mobile types of emission factors, thus in this paper we estimate the carbon emission of traffic and postal service sector using mobile type emission factors, and apply fixed type emission factors on other industries.

$$
C_{i}=\sum_{j=1}^{m} \sum_{k=1}^{n} C_{i j k}=\sum_{j=1}^{m} \sum_{k=1}^{n} E_{i j k} \times e f_{k}
$$

Then, combining formula (1) with expanding KAYA Identity to decompose $\mathrm{CO} 2$ per capita into intensity factors including Carbon Intensity and Energy Intensity, and structure factors including Energy Structure, Industrial Structure and Per Capita GDP.

$$
c_{i}=C_{i} / P_{i}=\sum_{j=1}^{m} \sum_{k=1}^{n} \frac{C_{i j k}}{E_{i j k}} \frac{E_{i j k}}{E_{i j}} \frac{E_{i j}}{Y_{i j}} \frac{Y_{i j}}{Y_{i}} \frac{Y_{i}}{P_{i}}=\sum_{j=1}^{m} \sum_{k=1}^{n} e f_{k} E S_{i j k} E I_{i j} I S_{i j} P G_{i}
$$

The meanings of variables in formula (1) and (2) are shown in Table1.

\begin{tabular}{|c|c|c|c|}
\hline Indicator & Meaning & Indicator & Meaning \\
\hline$C_{i}$ & $\mathrm{CO}_{2}$ emission of Region $i$ & $E S_{i j k}$ & $\begin{array}{l}\text { Energy Structure of Energy } k \text { of Sector } j \\
\text { in Region } i\end{array}$ \\
\hline$C_{i j k}$ & $\begin{array}{l}\mathrm{CO}_{2} \text { emission of Sector } j \text { in Region } i \\
\text { induced by Energy } k\end{array}$ & $E I_{i j}$ & Energy Intensity of Sector $j$ in Region $i$ \\
\hline$E_{i j k}$ & Usage of Energy $k$ of Sector $j$ in Region $i$ & $I S_{i j}$ & Industrial Structure of Sector $j$ in Region $i$ \\
\hline$E_{i j}$ & Usage of all Energy of Sector $j$ in Region $i$ & $P G_{i}$ & GDP per capita in Region $i$ \\
\hline$Y_{i j}$ & Added Value of Sector $j$ in Region $i$ & $e f_{k}$ & $\begin{array}{l}\mathrm{CO}_{2} \text { emission factor of Energy } k \text { (Carbon } \\
\text { Intensity of Energy } k \text { ) }\end{array}$ \\
\hline$Y_{i}$ & GDP in Region $i$ & $P_{i}$ & Populations in Region $i$ \\
\hline
\end{tabular}

Table 1. The meanings of indicators in formula (1) and (2)

By factor decomposition technique, Theil Index can measure overall regional inequities, also can dissect the inequities into contributions of factors. Specifically, the Theil Inequity Index of CO2 per capita of Region $i$ can be formulated as:

$$
T_{i}(c, p)=\sum_{a}\left(p_{a}^{i} \times \ln \frac{\bar{c}_{i}}{c_{i(a)}}\right)
$$

where $p_{a}^{i}=P_{a}^{i} / P_{i}$ indicates the population proportion of Province $a$ in Region $i, c_{i(a)}$ and $\bar{c}_{i}$ represents $\mathrm{CO} 2$ per capita of Province $a$ in Region $i$ and average CO2 per capita within Region $i$ respectively.

Based on Equation (2), taken emission factor as constant (Note 1), we can derive the Theil Inequity Index of 4 factors including Energy Structure, Energy Intensity, Industrial Structure and Per Capita GDP. To define each factor's Theil Inequity Index, we firstly define 4 virtual carbon emission factors $c_{\mathrm{i}}^{e s}, c_{\mathrm{i}}^{e i}, c_{\mathrm{i}}^{i s}$ and $c_{\mathrm{i}}^{p g}$ correspondingly. A certain virtual factor means the virtual $\mathrm{CO} 2$ per capita caused by the certain factor when taking other factors as constant, which are their average values. 


$$
\begin{aligned}
& c_{i}^{e s}=e s_{i} \times \overline{e t}_{i} \times \overline{s s}_{i} \times \overline{p g}_{i} \\
& c_{i}^{e i}=\overline{e s}_{i} \times e i_{i} \times \overline{i s}_{i} \times \overline{p g}_{i} \\
& \mathbf{c}_{\mathrm{i}}^{i s}=\overline{\boldsymbol{e s}}_{\boldsymbol{i}} \times \overline{\boldsymbol{e}}_{\boldsymbol{i}} \times \boldsymbol{i s _ { i }} \times \overline{\boldsymbol{p g}}_{\boldsymbol{i}} \\
& c_{i}^{p g}=\overline{e s}_{i} \times \overline{e l}_{i} \times \overline{\boldsymbol{i s}}_{i} \times \boldsymbol{p g} \boldsymbol{g}_{i}
\end{aligned}
$$

Where $\overline{e s}_{i}, \bar{e}_{i}, \overline{l s}_{i}$ and $\overline{p g}_{i}$ refer to average Energy Structure, average Energy Intensity, average Industrial Structure and average per capita GDP of provinces within Region $i$ respectivly.

Further, based on Theil Index in (3), each factor's Theil Inequity Index can be represented as:

$$
\begin{aligned}
& \mathrm{T}_{\mathrm{i}}(e s, p)=\sum_{a} p_{a}^{i} \times \ln \frac{\bar{c}_{i}}{c_{i(a)}^{e s}}=\sum_{a} p_{a}^{i} \times \ln \frac{\bar{c}_{i}}{e s_{i} \times \bar{e}_{i} \times \bar{s}_{i} \times \overline{p g} \bar{g}_{i}}=\sum_{a} p_{a}^{i} \times \ln \frac{\overline{e s}_{i}}{e s_{i(a)}} \\
& \mathbf{T}_{\mathbf{i}}(e i, p)=\sum_{a} p_{a}^{i} \times \ln \frac{\bar{c}_{i}}{c_{i(a)}^{i i}}=\sum_{a} p_{a}^{i} \times \ln \frac{\bar{c}_{i}}{\overline{e s}_{i} \times e i_{i} \times \bar{s}_{i} \times \overline{p g} \bar{g}_{i}}=\sum_{a} p_{a}^{i} \times \ln \frac{\overline{e s}}{e \bar{s}_{i(a)}} \\
& \mathrm{T}_{\mathrm{i}}(i s, \boldsymbol{p})=\sum_{a} p_{a}^{i} \times \ln \frac{\bar{c}_{i}}{\bar{c}_{i(a)}^{i s}}=\sum_{a} p_{a}^{i} \times \ln \frac{\bar{c}_{i}}{\overline{e s}_{i} \times \bar{e}_{i} \times i s_{i} \times \overline{p g}_{i}}=\sum_{a} p_{a}^{i} \times \ln \frac{\overline{e_{i}}}{\overline{i s}_{i(a)}} \\
& \mathrm{T}_{\mathrm{i}}(p g, p)=\sum_{a} p_{a}^{i} \times \ln \frac{\bar{c}_{i}}{c_{i(a)}^{p g}}=\sum_{a} p_{a}^{i} \times \ln \frac{\bar{c}_{i}}{\overline{e s}_{i} \times \bar{e}_{i} \times \bar{s}_{i} \times p g_{i}}=\sum_{a} p_{a}^{i} \times \ln \frac{\overline{e s}}{p g_{i(a)}}
\end{aligned}
$$

The summation the inequity index of each factor equals the regional inequity index, showing that this decomposition technique is complete.

$$
\begin{aligned}
& \mathbf{T}_{\mathbf{i}}(e s, \boldsymbol{p})+\mathbf{T}_{\mathbf{i}}(e i, p)+\mathbf{T}_{\mathbf{i}}(i s, p)+\mathbf{T}_{\mathbf{i}}(\boldsymbol{p g}, \boldsymbol{p}) \\
& =\sum_{a} p_{a}^{i} \times\left(\ln \frac{\bar{c}_{i}}{c_{i(a)}^{e s}}+\ln \frac{\bar{c}_{i}}{c_{i(a)}^{e i}}+\ln \frac{\bar{c}_{i}}{c_{i(a)}^{i s}}+\ln \frac{\bar{c}_{i}}{c_{i(a)}^{p g}}\right) \\
& =\sum_{a} p_{a}^{i} \times \ln \frac{\bar{c}_{i}^{4}}{c_{i(a)}^{e s} c_{i(a)}^{i(} \cdot c_{i(a)}^{i s} \cdot c_{i(a)}^{p g}}=\sum_{a} p_{a}^{i} \times \ln \frac{\bar{c}_{i}}{c_{i(a)}} \\
& =\mathbf{T}_{\mathbf{i}}(\boldsymbol{c}, \boldsymbol{p})
\end{aligned}
$$

Firstly, we divide 30 provinces in China into 8 regions (Note 2), which are Northeast Region (Liaoning Province, Jilin Province and Heilongjiang Province), Jingjin Region (Bejing City and Tianjin City), Northern Coastal Region (Hebei Province and Shandong Province), Eastern Coastal Region (Jiangsu Province, Shanghai City and Zhejiang Province), Southern Coastal Region (Fujian Province, Guangdong Province and Hainan Province), Central Region (Shanxi Province, Anhui Province, Jiangxi Province, Henan Province, Hunan Province and Hubei Province), Northwest Region (Neimenggu Province, Shaanxi Province, Gansu Province, Qinghai Province, Ningxia Province and Xinjiang Province) and Southwest Region (Guangxi Province, Chongqing City, Sichuan Province, Guizhou Province and Yunnan Province) respectively.

Then, based on the Energy Balance Table from China Energy Statistical Yearbook, we investigate 6 sectors, which are Agriculture Sector, Industry Sector, Construction Sector, Transportation and Post Sector, Wholesale/Retail and Hotel/Catering Sector, and Other Service Sector. The carbon emission induced by residential consumption accounts for less than $5 \%$ of total emission (Note 3), thus we only consider the $\mathrm{CO}_{2}$ from industrial production.

Last but not least, we consider 8 types of energy emitting $\mathrm{CO}_{2}$, which are Raw Coal, Coke, Crude Oil, Gasoline, Kerosene, Diesel, Fuel Oil and Natural Gas. To consider full effects of energy using and avoid double accounting, we add the input of transformation to the final consumption of Industry Sector, and subtract non-energy use (Note 4).

According to Equation (2), three dimensions (Region, Sector and Energy) embodied in the decomposition of per capita $\mathrm{CO}_{2}$ inequity. However, in Equation (8) - (11), we only one dimension (Region) when calculating factor inequity index. To fix this problem technically, for the reason that Industry Sector (Note 5) and Raw Coal/Coke (Note 6) are the sector and energy types making the predominant contributions when emitting $\mathrm{CO}_{2}$, in this paper, the influential factors are defined as follows: (1) Regional Energy Structure is defined as the proportion of Raw Coal and Coke consumption in total energy use, in terms of standard coal. (2) Regional Energy Intensity is defined as the amount of standard coal consumptions per unit of GDP, (3) Regional Industrial Structure is defined as the added value of Industrial Sector in regional GDP, (4) we lastly define Regional Per Capita Output 
as regional per capita GDP.

The sector added value, GDP and population data are from China Statistical Yearbook, energy usage data are from China Energy Statistical Yearbook. We use interpolation method to estimate missing data. The time period starts from Year 1997 to Year 2014.

\section{Results}

\subsection{Carbon Emissions and Energy Use}

According to the Equation (1), China's eight regional carbon dioxide emissions can be estimated, as shown in Figure (1).

From the point of view of total carbon dioxide emissions, during the period of 1997-2014, $\mathrm{China}^{\text {'s } \mathrm{CO}_{2}}$ emissions rise from 3.05 billion tons to 9.09 billion tons, at an average annual growth rate of 6.64\%. Among the 8 regions, the largest contributions are central region, northwest and north coastal area, contributing 1.98 billion tons, 1.53 billion tons and 1.52 billion tons respectively in 2014. Jingjin Region makes the smallest contribution, which is 0.24 billion tons in 2014. Between 1997 and 2014, Northeast China, Jingjin Region, Northern Coast, Eastern Coast, Southern Coast, Central Region, Northwest and Southwest Region contributions 10.43\%, 2.61\%, $16.74 \%, 12.63 \%, 7.81 \%, 21.81 \%, 16.79 \%$ and $11.18 \%$ of $\mathrm{CO}_{2}$ emissions on average. Among them, the biggest contributions are Central Region, Northern Coastal and Northwest Region, they account for more than 55.34\% carbon dioxide in total.

From the point of view of the trend of $\mathrm{CO}_{2}$ emissions, according to the characteristics of carbon emission growth rate, 1997-2014 can be divided into three stages: 1) the first is 5-year "moderate" growth period from 1997 to 2002: in this stage, $\mathrm{CO}_{2}$ emissions present a slow annual growth rate of $3.11 \%$ on average, companied with medium speed of the economy growing since the reform and opening in China. 2) then followed by another 5-year "high speed" growth period from 2002-2007: in the stage, under the circumstance of strong economic growth since China joining WTO, $\mathrm{CO}_{2}$ emissions grows at an annual rate of $14.18 \%$ averagely; 3 ) lastly comes the "medium" growth period from 2007-2014: affected by financial crisis and economic structure transformation, China's economic growth has slowed recently, the average annual growth rate of $\mathrm{CO}_{2}$ emissions dropped to $6.28 \%$ as a consequence.

Similar to $\mathrm{CO}_{2}$, regional per capita $\mathrm{CO}_{2}$ also presents the "three-stage" growth characteristic (as shown in Figure 2): average annual growth rate of China's per capita $\mathrm{CO}_{2}$ in $1997-2002$ is $2.76 \%$, then increases to $14.00 \%$ in 2002-2007, followed by a falling back to 5.39\% in 2007-2014. Among regions, during 1997-2014, the per capita $\mathrm{CO}_{2}$ of Northeast Region, Jingjin Region, Northern Coast and Southwest Region respectively is per capita 7.50 tons, 6.09 tons, 7.84 tons and 9.59 tons, above national levels (5.90 tons per capita). That of Eastern Coast, Southern Coast, Central Region, and Northwest Region is lower than national average level, which is per capita 5.80 tons, 3.61 tons, 5.83 tons and 3.53 tons respectively.

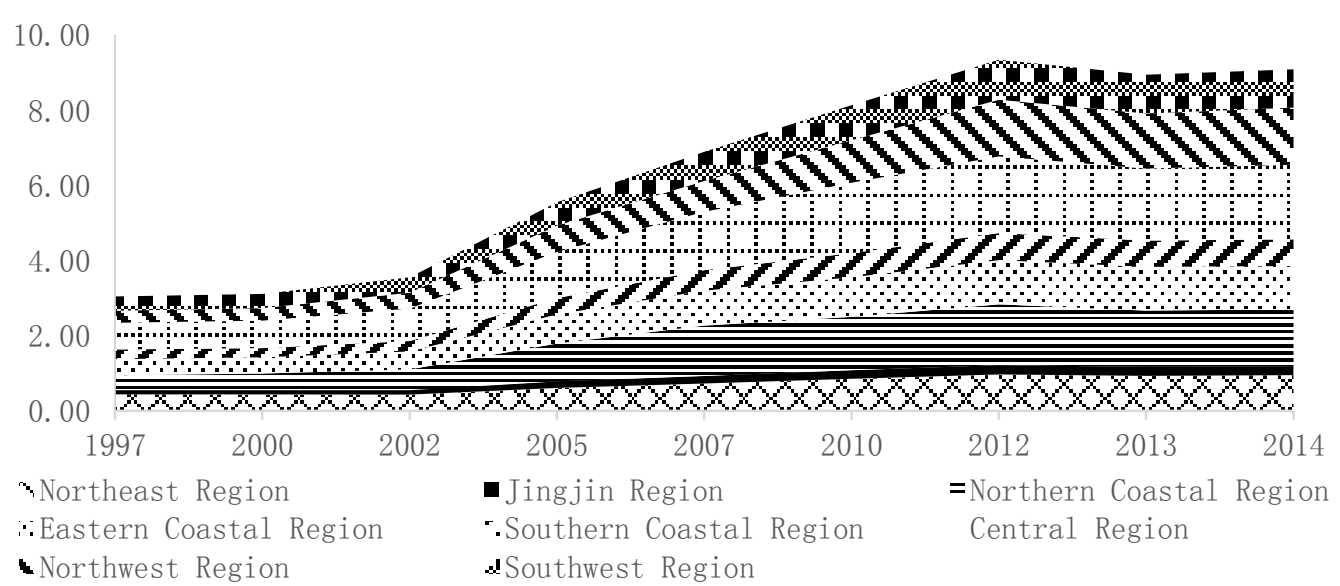

Figure1. The total carbon emission of 8 regions in China from 1997-2014 (billion tons) 


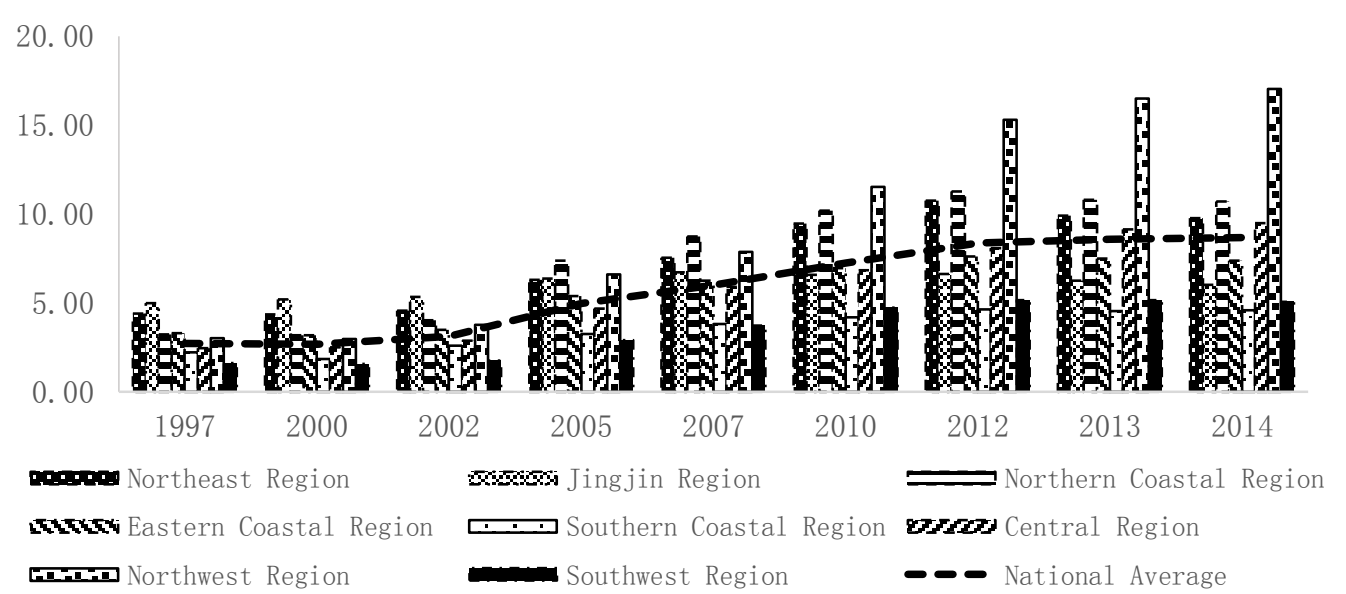

Figure2. The per capita $\mathrm{CO}_{2}$ emission of 8 regions in China from 1997-2014 (tons/person)

Generally speaking, the largest amount of $\mathrm{CO}_{2}$ emission exists in the Central and Northern Coast, whereas Northwest Region possesses the highest per capita $\mathrm{CO}_{2}$ levels, and its contribution rate is rising year by year.

As the support for economic growth, the total energy consumption is the direct cause of $\mathrm{CO}_{2}$ emission, regional differences in energy use can partly explain the diverse $\mathrm{CO}_{2}$ emissions. Behind the growth of carbon dioxide emissions is the high energy consumptions, China's energy consumptions in terms of standard coal increase from 1.25 billion tce in 1997 to 4.47 billion tce in 2014, the average annual growth is $7.23 \%$, similar to that of $\mathrm{CO}_{2}$ growth rate.

China's provincial $\mathrm{CO}_{2}$ emissions and energy consumptions during 1997-2014 are shown in Figure 3. The average annual growth rate of the $\mathrm{CO}_{2}$ emissions in Northeast, Jingjin Region, Northern Coast, Eastern Coast, Southern Coast, Central Region, Northwest and Southwest Region is 5.13\%, 4.33\%, 8.00\%, 6.12\%, 6.37\%, $8.67 \%, 11.44 \%$ and $7.17 \%$, and that of total energy consumptions is $5.03 \%, 4.62 \%, 7.99 \%, 6.09 \%, 6.22 \%$, $8.72 \%, 11.41 \%$ and $7.23 \%$, their consistency shows that $\mathrm{CO}_{2}$ emissions and energy usages are tightly correlated. Among them, the growth rate of Northwest Region is the highest, the lowest is Beijing and Tianjin area.

Comparing China's eight regional $\mathrm{CO}_{2}$ emissions and energy consumptions, the Northern Coast, Central Region and Northwest China share the highest proportion of both the carbon emission and energy consumption, those three areas' $\mathrm{CO}_{2}$ emissions and energy usages account for $58.55 \%$ and $57.74 \%$ respectively during $1997-2014$, contributing more than a half countrywide.

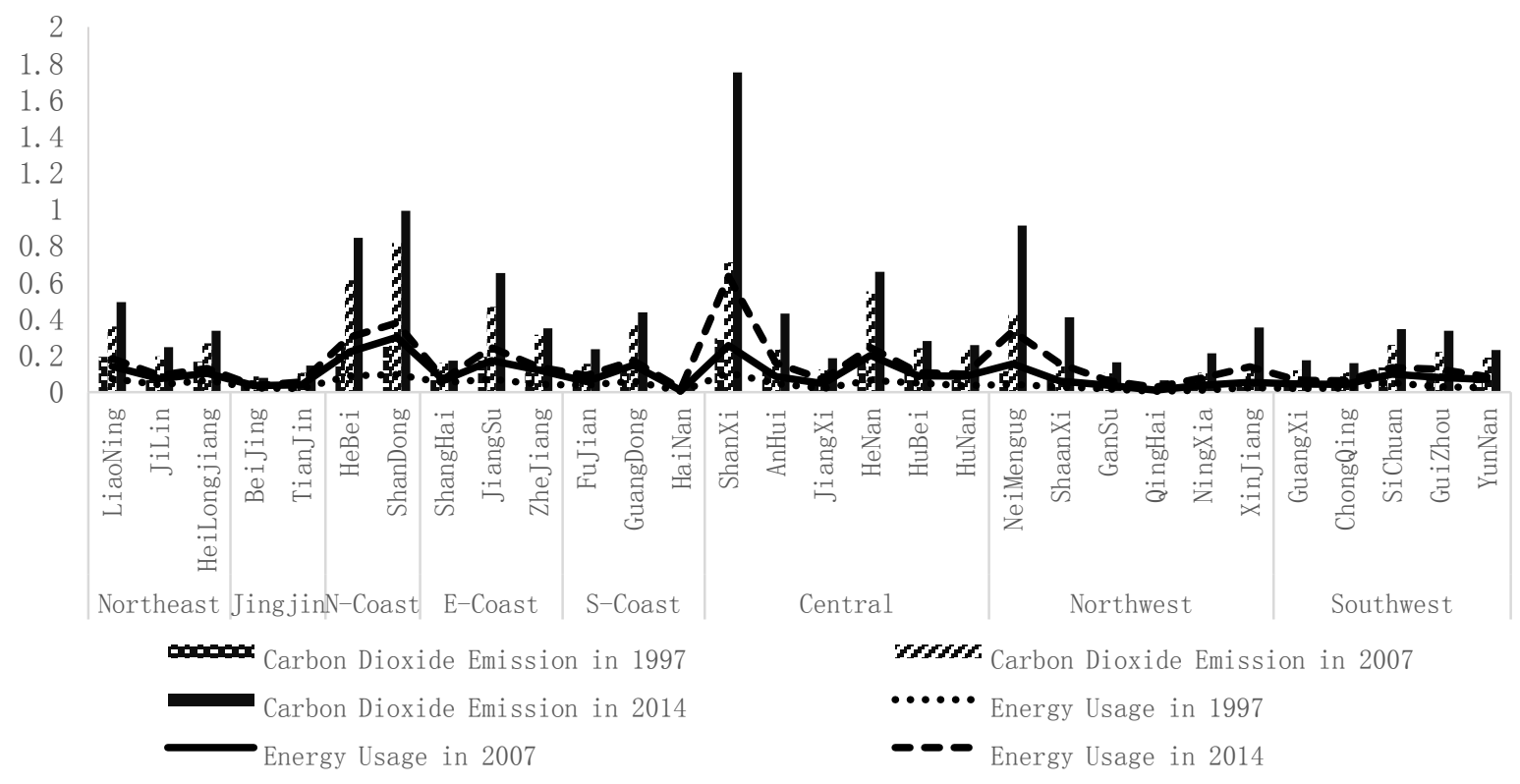

Figure 3. The provincial $\mathrm{CO}_{2}$ and energy usage in 8 regions of China in 1997-2014 (billion tons) 


\subsection{Inter-Regional Inequity of Per Capita CO2}

This paper combines Theil Index and expanding KAYA decomposition technique, builds a model for regional inequality of $\mathrm{CO} 2$ emissions per capita, and decomposes per capita $\mathrm{CO} 2$ into factor inequity index, namely the energy efficiency inequity indexes (Energy Structure inequity index and Energy Intensity inequity index) and the economic efficiency inequity indexes (Industrial Structure inequity index and Per Capita Output inequity index). Based on equation (8) to (11) thought, this paper estimates China 8 inter- and intra- regional inequalities of per capita CO2. The results are shown in Table (2) - Table(4).

Table 2. The Theil index of per capita $\mathrm{CO}_{2}$ emission in China from 1997-2014 (Overall)

\begin{tabular}{lccccccccc}
\hline & 1997 & 2000 & 2002 & 2005 & 2007 & 2010 & 2012 & 2013 & 2014 \\
\hline $\mathrm{T}(\mathrm{es}, \mathrm{p})$ & -0.044 & -0.027 & -0.020 & -0.025 & -0.025 & -0.008 & 0.006 & 0.007 & 0.048 \\
$\mathrm{~T}(\mathrm{ei}, \mathrm{p})$ & 0.332 & 0.412 & 0.514 & 0.421 & 0.454 & 0.526 & 0.612 & 0.770 & 0.764 \\
$\mathrm{~T}(\mathrm{is}, \mathrm{p})$ & -0.052 & -0.035 & -0.025 & -0.020 & -0.011 & -0.027 & -0.028 & -0.032 & -0.034 \\
$\mathrm{~T}(\mathrm{gp}, \mathrm{p})$ & 0.092 & 0.077 & 0.041 & -0.002 & -0.034 & -0.032 & -0.026 & -0.021 & -0.033 \\
$\mathrm{~T}(\mathrm{c}, \mathrm{p})$ & 0.328 & 0.427 & 0.510 & 0.374 & 0.383 & 0.459 & 0.565 & 0.724 & 0.745 \\
\hline
\end{tabular}

Table 3. The Theil index of per capita $\mathrm{CO}_{2}$ emission in China from 1997-2014 (Inter-regional)

\begin{tabular}{lllllllllll}
\hline & 1997 & 2000 & 2002 & 2005 & 2007 & 2010 & 2012 & 2013 & 2014 \\
\hline $\mathrm{T}(\mathrm{es}, \mathrm{p})$ & 0.007 & 0.010 & 0.010 & 0.009 & 0.013 & 0.014 & 0.016 & 0.018 & 0.022 \\
$\mathrm{~T}(\mathrm{ei}, \mathrm{p})$ & 0.005 & 0.011 & 0.013 & 0.012 & 0.014 & 0.026 & 0.038 & 0.049 & 0.054 \\
$\mathrm{~T}(\mathrm{is}, \mathrm{p})$ & 0.012 & 0.008 & 0.002 & 0.004 & -0.008 & -0.019 & -0.020 & -0.020 & -0.018 \\
$\mathrm{~T}(\mathrm{gp}, \mathrm{p})$ & -0.017 & -0.019 & -0.019 & -0.019 & -0.020 & -0.021 & -0.021 & -0.021 & -0.021 \\
$\mathrm{~T}(\mathrm{c}, \mathrm{p})$ & 0.007 & 0.010 & 0.006 & 0.006 & -0.001 & 0.000 & 0.013 & 0.026 & 0.037 \\
\hline
\end{tabular}

The overall inequity index is the aggregation of inter-regional inequity and intra-regional inequity, reflecting the inequality of per capita $\mathrm{CO}_{2}$ emissions in China. According to Table 2, the inequity of per capita $\mathrm{CO}_{2}$ in China also presents three-stage characteristics, but its changing trend is inverse to that of carbon dioxide per capita: 1) In 1997-2002 "moderate" growth stage, the inequality "expansions", increasing from 0.328 to 0.510 , with an annual growth rate of $9.22 \%$; 2) In the "high speed" growth stage in 2002-2007, the inequality "shrinks", declining from 0.510 to 0.383 , with an average annual growth rate of $-5.55 \%$, narrowing the inequality; 3 ) The "Medium" growth stage during 2007-2014 witnesses the inequality of $\mathrm{CO}_{2}$ per capita rising again from 0.383 to 0.745 , by an annual growth rate of $9.96 \%$. In this phase, inequality "expansions again".

That is to say, when the per capita $\mathrm{CO}_{2}$ has higher growth, its inequality decreases. Conversely, when the growth of $\mathrm{CO}_{2}$ per capita is slowing, its inequality increases instead.

Among the 4 factors, energy intensity is increasing inequality, its inequity index is 0.764 in 2014, contributing $102.5 \%$ of the total inequity. Industrial structure is a factor reducing the inequality, its Theil Inequity Index in 2014 is 0.034 , accounting for $4.5 \%$. Energy structure factor has a turning point in 2012 , turning from an inequity decreasing factor (negative value in inequity index) into an inequity increasing one (positive value in inequity index), with its inequity value rising from -0.073 in 1997 to 0.006 in 2012, then to 0.048 in 2014 . On the contrary, per capita output factor has a turning point in 2005, but in an opposite way, gradually shifting from an inequity expanding factor to an inequity shirking one. Its inequity value drops from 0.092 in 1997 to -0.002 in 2005, then further to -0.033 in 2014.

The inter-regional inequality of $\mathrm{CO}_{2}$ per capita also displays the trend of rising-falling-rising, as shown in Table 3 and Figure 4. Year 2007 is a turning point, before that the inter-regional inequality falls from 0.007 to -0.001 , achieving an extremely low point, then rises to 0.037 after that. In other words, the inter-regional inequity of per capita carbon dioxide is closing during 1997-2007, but in 2007-2014 the inequity is expanding.

Factors have different effects on the inter-regional inequality. Energy intensity and energy structure factors, on behalf of regional differentiations in energy efficiency, constantly enlarge inter-regional inequality of per capita $\mathrm{CO}_{2}$. Per capita output factor always reduces the inequality, and the industrial structure factor has a positive-then-negative effect before and after 2007. Energy Intensity Factor is the biggest positive influential factor, showing a trend of obvious rising, up from 0.005 in 1997 to 0.054 in 2014, and its contribution ratio is $146.70 \%$ to total inter-regional inequity of per capita carbon dioxide in 2014. That means regional difference in energy intensity is the main reason for the regional differences in per capita $\mathrm{CO}_{2}$ emissions. Energy Structure 
Factor's inequity index is slowly rising, increasing from 0.007 in 1997 to 0.022 in 2014, the contribution rate is $59.34 \%$ in 2014 . The differences among regional energy structures partly explain the inequality of regional per capita $\mathrm{CO}_{2}$. Per Capita Output, on the other hand, is a negative factor reducing the inequality of regional $\mathrm{CO}_{2}$ emissions per capita on the whole. Its index value is basically sTable, starting from -0.017 in 1997 and staying constant since 2010 at -0.021 , and its contribution rate in 2014 is $56.87 \%$. As the biggest negative influential factor, it shows that convergence effect exists in national economic developments, as a result narrowing the regional differences of $\mathrm{CO}_{2}$ emissions per capita. Industrial Structure Factor's inequity index declines year by year, from 0.012 in 1997, presenting negative effects since 2007, continued declining to -0.018 in 2014, contributing $-49.17 \%$. Rebalancing of regional industrial structure narrows the regional inequality of $\mathrm{CO}_{2}$ emissions per capita.

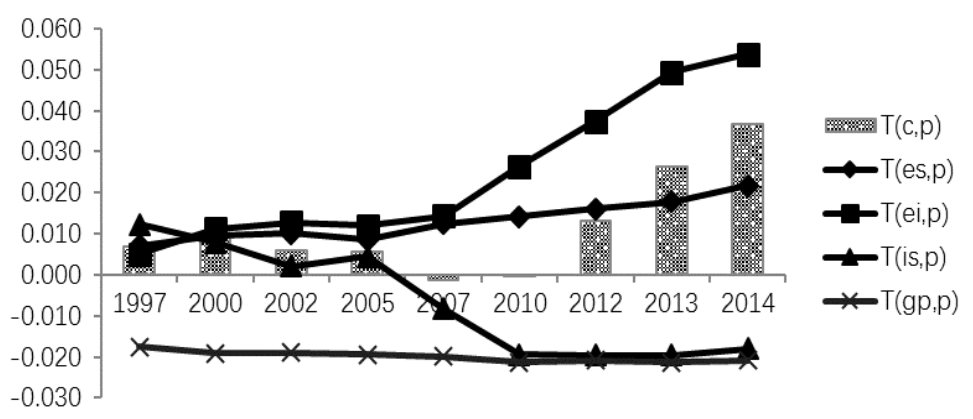

Figure 4. The Theil index of per capita $\mathrm{CO}_{2}$ emission in China from 1997-2014 (Inter-regional)

Factors have different effects on the inter-regional inequality. Energy intensity and energy structure factors, on behalf of regional differentiations in energy efficiency, constantly enlarge inter-regional inequality of per capita $\mathrm{CO}_{2}$. Per capita output factor always reduces the inequality, and the industrial structure factor has a positive-then-negative effect before and after 2007. Energy Intensity Factor is the biggest positive influential factor, showing a trend of obvious rising, up from 0.005 in 1997 to 0.054 in 2014, and its contribution ratio is $146.70 \%$ to total inter-regional inequity of per capita carbon dioxide in 2014. That means regional difference in energy intensity is the main reason for the regional differences in per capita $\mathrm{CO}_{2}$ emissions. Energy Structure Factor's inequity index is slowly rising, increasing from 0.007 in 1997 to 0.022 in 2014, the contribution rate is $59.34 \%$ in 2014 . The differences among regional energy structures partly explain the inequality of regional per capita $\mathrm{CO}_{2}$. Per Capita Output, on the other hand, is a negative factor reducing the inequality of regional $\mathrm{CO}_{2}$ emissions per capita on the whole. Its index value is basically sTable, starting from -0.017 in 1997 and staying constant since 2010 at -0.021 , and its contribution rate in 2014 is $56.87 \%$. As the biggest negative influential factor, it shows that convergence effect exists in national economic developments, as a result narrowing the regional differences of $\mathrm{CO}_{2}$ emissions per capita. Industrial Structure Factor's inequity index declines year by year, from 0.012 in 1997, presenting negative effects since 2007, continued declining to -0.018 in 2014, contributing $-49.17 \%$. Rebalancing of regional industrial structure narrows the regional inequality of $\mathrm{CO}_{2}$ emissions per capita.

Next, we'll focus on the 4 factors' differences and trends among 8 regions.

Energy structure represents the proportion of raw coal and coke in total energy use. These two kinds of energy have relatively large portions and low efficiency, can be representative samples for energy structure. In general, the use of coal and coke in the energy structure falls from $83.28 \%$ in 1997 to $82.57 \%$ in 2005 , with an average annual decreasing rate of $0.11 \%$. Then it rises at a rate of $0.23 \%$ to $84.26 \%$ in 2014 .

We also can conclude from Figure 5 that, less developed regions like Central and Northern Coastal areas have higher coal and coke use ratio than the national average level, whereas that of more developed districts including Beijing-Tianjin region, Southern Coastal and Eastern Coastal Region is below national average, and coal use ratios of the rest regions fluctuate around the average. During 1997-2014, the range of 8 regions' energy structures has widened, where Central Region has the highest energy structure, its gap between the lowest region in 1997 (Northern Coastal Region) is 29.84\%, and expands to 34.21\% comparing to Jingjin District in 2014.

Data shows that in China, less developed areas tend to have higher coal ratio, while the proportion of coal and coke use in developed areas is relatively low, and the gap is being widened between these two groups. 


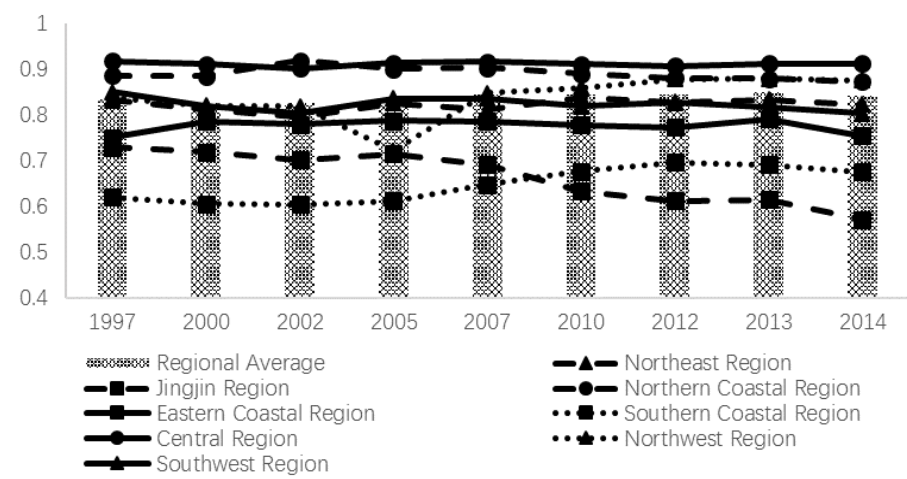

Figure 5. The energy structures of 8 regions in China from 1997-2014

Energy Intensity represents the energy efficiency of energy use. As shown in Figure 6, in 1997-2014, China's energy intensity declines at an average rate of 2.84\%, falling from 1.63 (Note 7) in 1997 to 1.00 in 2014. According to energy intensity, 8 regions can be divided into three categories: high energy intensity area (Northwest), medium energy intensity area (Central, North Coastal, Northeast and Southwest) and low energy intensity area (Jingjin, Eastern Coastal and Southern Coastal Region). It can be seen that an inverse relationship exists between energy intensity and economic development level, and the regional differentiations change over time. In 1997, regional differences of each other's energy intensity is relatively uniform. From 2000, 8 regions begin to differentiate into three groups, and regions gather within groups. Meanwhile, groups disperse gradually, and an obvious separation between groups has emerged since 2007.

Although the aggregation degree of energy intensity within groups declines, the differences between these three categories continues to expand. The difference of energy intensity between high energy intensity area and low energy intensity area in 1997 is 1.56 , then rises to 2.06 in 2014 . The growing of regional energy intensity differentiations is the cause of the expanding of energy intensity factor inequity index.

We use the ratio of added value of industry sector in GDP to represent the industrial structure. Industry sector accounts for about $90 \%$ (Note 8) of $\mathrm{CO}_{2}$ emissions, rising year by year, thus its proportion can simulates the characteristics of regional industrial structure. The industrial structure in China stays above $20 \%$ before 2000 (Figure 7), and then falls to $17.98 \%$ in 2014 at an average declining rate of $1.51 \%$, showing that the proportion of industry sector in China has fallen.

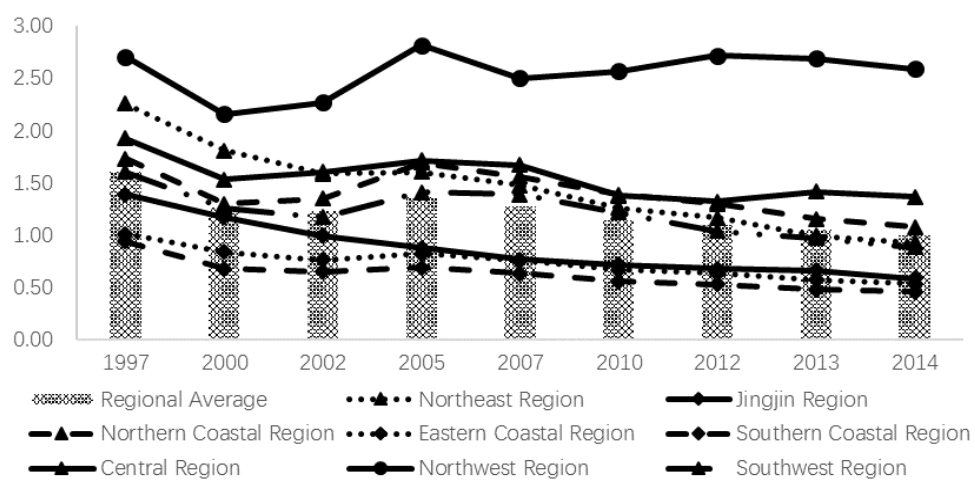

Figure 6. The energy intensity of 8 regions in China from 1997-2014 (tce/ten thousand yuan)

Among 8 regions, the industry structures in Northern Coastal, Northeast and Southern Coastal in 1997-2014 are always higher than the national average level, whereas that of Jingjin Region and the Southwest are always lower than the average; Eastern Coast is -above-then-below, while the Central and Northwestern are below-then-above average level before and after 2007. In addition, industrial structures among 8 regions changes over time. In 1997, differences of each region's industrial structure are more evenly, with a range of 0.10 between the highest (Eastern Coastal Region) and the lowest (Jingjin Region); In 2014, Jingjin Region's industrial structure stays below the national average level, and the other seven areas are close to or above the average. Except for Beijing-Tianjin region, the remaining seven regions form a high-industrial-structure group. 
Within the group, the level of regional industrial structures gradually closes up, and extreme difference is 0.05 within groups. However, the industrial structure difference between Jingjin Region and the other seven regions(average) is 0.10 . The expanding disparity causes industrial structure factor index being negative, and the value is enlarged.

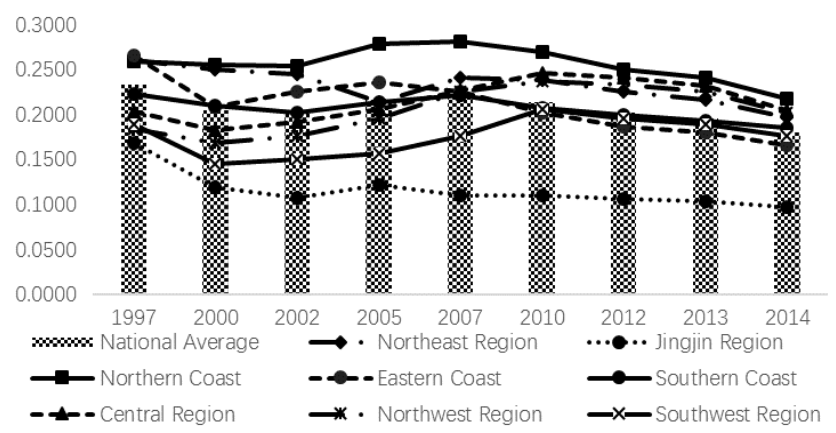

Figure 7. The industry structure of 8 regions in China from 1997-2014

Per capita output represents the level of economic development. We use comparable price to calculate the per capita GDP. In China from 1997-2014, per capita GDP grows at an average rate of 10.15\%, and reaches 32538.88 yuan per capita in 2014.

It can be seen from the Figure 8 that, according to whether the output per head is higher than the national average level or not, 8 regions can be distinguished into two categories: high per capita output areas (Eastern Coast, Jingjin region, Northern Coastal and Southern Coastal Region) and low per capita output area (the Northeast, Southwest, Northwest and Central region). Moreover, the gap between these two categories has been widened between, the average per capita output gap expanded from 5696.89 yuan per head in 1997 to 18349.73 yuan per head in 2014. In particular, after 2010, because of its higher level of per capita output, the Eastern Coastal area has a trend of separation from the high per capita output category, being the highest one. And similar to the industrial structure factor, the increasing of the difference between categories leads to inequity index of per capita output factor being negative and expanding.

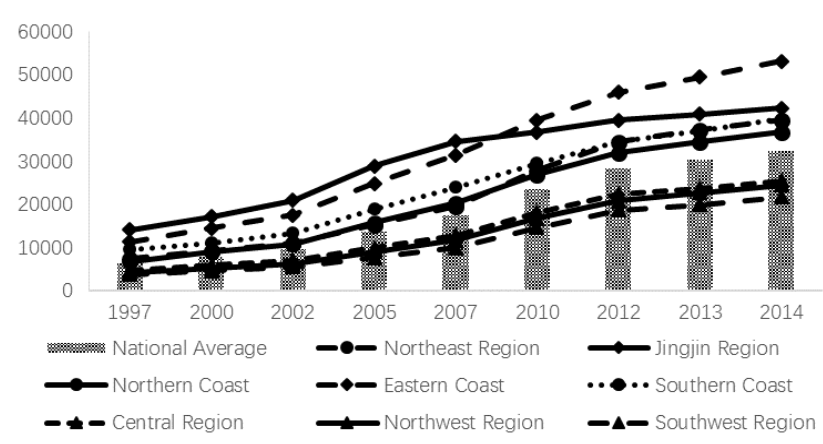

Figure 8. The per capita GDP of 8 regions in China from 1997-2014 (yuan)

\subsection{Intra-Regional Inequity of Per Capita $\mathrm{CO}_{2}$}

The per capita $\mathrm{CO}_{2}$ emissions inequalities within regions also have different characteristics. The intra-regional inequality of $\mathrm{CO}_{2}$ emissions per capita is shown in Table 4.

Overall, the intra-regional inequity of per capita $\mathrm{CO}_{2}$ emission differs among regions. Specifically, the inequity within Northeast Region, Jingjin Region, Northern Coast, Central Region, Northwest and Southwest Region has an upward trend, with an average annual growth rate of $16.05 \%, 10.03 \%, 1.64 \%, 4.24 \%, 4.43 \%$ and $1.94 \%$ respectively. The Eastern Coast and Southern Coastal Region show a decline trend, with an average annual declining rate of $192.92 \%$ and $5.75 \%$. In 2014, ranking by the regional contributions from large to small, Beijing-tianjin region, Northwest, Southwest, Northern Coast, Northeast, Eastern Coastal and Southern Coastal contributes $45.19 \%, 25.70 \%, 17.39 \%, 14.80 \%, 4.37 \%, 2.89 \%, 5.61 \%$ and $9.64 \%$ respectively.

Among the 8 regions, less developed regions including the Central, Jingjin, Northwest and Southwest are 
relatively high, whose regional inequities are relatively high, compared to more developed regions such as Southern Coastal Region and Eastern Coastal Region (negative contributions in 2014) and Northern Coastal Region and Northeast Region (small positive contributions).

Regions also have various trends of carbon inequality. The $\mathrm{CO}_{2}$ emissions per capita inequality of Central Region is in accordance with the national trend, which presents the rise-fall-rise three phase characteristics. Inequalities in Eastern Coast and Southern Coast reduce year by year, and are all being negative since 2010 began to reduce this inequality. Jingjin Region rises its inequity year by year. The inequality trends of rest regions are relatively sTable.

Among the 4 influencing factors, Energy Structure (ES), Energy Intensity (EI), Industrial Structure (IS) and Per capita GDP (PG) each affects the regional inequality of $\mathrm{CO}_{2}$ emissions per capita differently across regions. Also, each region has different main influencing factors and directions, as shown in Table 5. To be specific, 1) Energy Structure Factor affects more developed regions negtively, including Jingjin Region and Eastern Coastal region. 2) Energy Intensity Factor affects less developed regions positively, including the Northeast, Northern Coast, the Southern Coastal, the Central, Northwest and Southwest region. 3) Industry Structure (IS) has a significant positive effect on Northeast China and the Beijing-tianjin area, but has a negative effect on Southern Coast and Northwest Region. 4) Per capita GDP (PG) only has a negative effect on the Northern Coastal (Table 6).

Table 4. The Theil index of per capita $\mathrm{CO}_{2}$ emission in China from 1997-2014 (Intra-regional)

\begin{tabular}{|c|c|c|c|c|c|c|c|c|c|c|}
\hline & & 1997 & 2000 & 2002 & 2005 & 2007 & 2010 & 2012 & 2013 & 2014 \\
\hline \multirow{5}{*}{ North-east } & $\mathrm{T}(\mathrm{es}, \mathrm{p})$ & 0.003 & 0.003 & 0.005 & 0.000 & 0.000 & 0.003 & 0.005 & 0.003 & 0.003 \\
\hline & $\mathrm{T}(\mathrm{ei}, \mathrm{p})$ & 0.011 & -0.001 & 0.001 & 0.009 & 0.011 & 0.007 & 0.008 & 0.005 & 0.005 \\
\hline & $\mathrm{T}(\mathrm{is}, \mathrm{p})$ & -0.005 & -0.005 & -0.004 & -0.003 & -0.003 & 0.000 & 0.003 & 0.005 & 0.010 \\
\hline & $\mathrm{T}(\mathrm{gp}, \mathrm{p})$ & -0.007 & -0.005 & -0.005 & -0.002 & -0.003 & 0.000 & 0.001 & 0.003 & 0.003 \\
\hline & $\mathrm{T}(\mathrm{c}, \mathrm{p})$ & 0.002 & -0.008 & -0.003 & 0.002 & 0.005 & 0.011 & 0.018 & 0.016 & 0.022 \\
\hline \multirow{5}{*}{ Jing-jin } & $\mathrm{T}(\mathrm{es}, \mathrm{p})$ & 0.001 & 0.000 & 0.006 & 0.008 & 0.016 & 0.036 & 0.053 & 0.051 & 0.085 \\
\hline & $\mathrm{T}(\mathrm{ei}, \mathrm{p})$ & 0.015 & 0.016 & 0.020 & 0.023 & 0.022 & 0.020 & 0.022 & 0.013 & 0.021 \\
\hline & $\mathrm{T}(\mathrm{is}, \mathrm{p})$ & 0.026 & 0.043 & 0.060 & 0.059 & 0.083 & 0.083 & 0.086 & 0.083 & 0.084 \\
\hline & $\mathrm{T}(\mathrm{gp}, \mathrm{p})$ & -0.004 & -0.005 & -0.006 & -0.007 & -0.008 & -0.001 & 0.002 & 0.002 & 0.001 \\
\hline & $\mathrm{T}(\mathrm{c}, \mathrm{p})$ & 0.038 & 0.054 & 0.080 & 0.083 & 0.114 & 0.138 & 0.163 & 0.149 & 0.191 \\
\hline \multirow{5}{*}{$\begin{array}{l}\text { Northern } \\
\text { Coast }\end{array}$} & $\mathrm{T}(\mathrm{es}, \mathrm{p})$ & 0.003 & 0.004 & 0.001 & 0.003 & 0.002 & 0.003 & 0.004 & 0.004 & 0.005 \\
\hline & $\mathrm{T}(\mathrm{ei}, \mathrm{p})$ & 0.026 & 0.041 & 0.031 & 0.017 & 0.016 & 0.023 & 0.026 & 0.034 & 0.029 \\
\hline & $\mathrm{T}(\mathrm{is}, \mathrm{p})$ & 0.000 & 0.000 & -0.001 & -0.003 & -0.002 & -0.001 & 0.001 & 0.002 & 0.002 \\
\hline & $\mathrm{T}(\mathrm{gp}, \mathrm{p})$ & -0.004 & -0.004 & -0.005 & -0.005 & -0.005 & -0.004 & -0.004 & -0.004 & -0.003 \\
\hline & $\mathrm{T}(\mathrm{c}, \mathrm{p})$ & 0.025 & 0.040 & 0.027 & 0.012 & 0.012 & 0.022 & 0.027 & 0.036 & 0.033 \\
\hline \multirow{5}{*}{ Eastern Coast } & $\mathrm{T}(\mathrm{es}, \mathrm{p})$ & -0.013 & -0.016 & -0.018 & -0.027 & -0.029 & -0.027 & -0.028 & -0.030 & -0.030 \\
\hline & T(ei,p) & 0.002 & 0.008 & 0.006 & -0.014 & -0.016 & -0.008 & -0.012 & -0.006 & -0.011 \\
\hline & $\mathrm{T}(\mathrm{is}, \mathrm{p})$ & 0.001 & -0.002 & -0.010 & -0.010 & -0.011 & -0.014 & -0.015 & -0.017 & -0.017 \\
\hline & $\mathrm{T}(\mathrm{gp}, \mathrm{p})$ & 0.156 & 0.141 & 0.109 & 0.080 & 0.060 & 0.033 & 0.018 & 0.017 & 0.015 \\
\hline & $\mathrm{T}(\mathrm{c}, \mathrm{p})$ & 0.146 & 0.132 & 0.087 & 0.030 & 0.003 & -0.015 & -0.036 & -0.035 & -0.042 \\
\hline \multirow{5}{*}{$\begin{array}{l}\text { Southern } \\
\text { Coast }\end{array}$} & $\mathrm{T}(\mathrm{es}, \mathrm{p})$ & -0.050 & -0.035 & -0.033 & -0.032 & -0.035 & -0.049 & -0.050 & -0.050 & -0.049 \\
\hline & $\mathrm{T}(\mathrm{ei}, \mathrm{p})$ & 0.007 & -0.004 & 0.033 & 0.032 & 0.040 & 0.068 & 0.104 & 0.108 & 0.104 \\
\hline & $\mathrm{T}(\mathrm{is}, \mathrm{p})$ & -0.088 & -0.090 & -0.067 & -0.078 & -0.071 & -0.075 & -0.076 & -0.075 & -0.080 \\
\hline & $\mathrm{T}(\mathrm{gp}, \mathrm{p})$ & -0.066 & -0.060 & -0.063 & -0.081 & -0.079 & -0.068 & -0.051 & -0.050 & -0.047 \\
\hline & $\mathrm{T}(\mathrm{c}, \mathrm{p})$ & -0.197 & -0.189 & -0.129 & -0.159 & -0.145 & -0.124 & -0.074 & -0.066 & -0.072 \\
\hline \multirow{5}{*}{ Central } & $\mathrm{T}(\mathrm{es}, \mathrm{p})$ & 0.003 & 0.002 & 0.004 & 0.001 & 0.001 & 0.000 & 0.001 & 0.003 & 0.003 \\
\hline & $\mathrm{T}(\mathrm{ei}, \mathrm{p})$ & 0.159 & 0.165 & 0.174 & 0.142 & 0.145 & 0.155 & 0.183 & 0.328 & 0.329 \\
\hline & $\mathrm{T}(\mathrm{is}, \mathrm{p})$ & 0.002 & -0.001 & 0.001 & 0.006 & 0.007 & 0.002 & -0.001 & -0.002 & -0.001 \\
\hline & $\mathrm{T}(\mathrm{gp}, \mathrm{p})$ & 0.003 & 0.001 & 0.005 & 0.004 & 0.002 & 0.001 & 0.003 & 0.009 & 0.007 \\
\hline & $\mathrm{T}(\mathrm{c}, \mathrm{p})$ & 0.166 & 0.168 & 0.183 & 0.153 & 0.155 & 0.158 & 0.186 & 0.338 & 0.337 \\
\hline \multirow{5}{*}{ North-west } & $\mathrm{T}(\mathrm{es}, \mathrm{p})$ & -0.002 & -0.005 & -0.004 & 0.008 & 0.001 & -0.001 & -0.002 & -0.001 & -0.002 \\
\hline & $\mathrm{T}(\mathrm{ei}, \mathrm{p})$ & 0.053 & 0.091 & 0.169 & 0.107 & 0.123 & 0.140 & 0.144 & 0.131 & 0.128 \\
\hline & $\mathrm{T}(\mathrm{is}, \mathrm{p})$ & -0.011 & -0.006 & -0.012 & -0.005 & -0.004 & -0.001 & -0.002 & -0.003 & -0.005 \\
\hline & $\mathrm{T}(\mathrm{gp}, \mathrm{p})$ & 0.021 & 0.013 & 0.012 & 0.011 & 0.014 & 0.015 & 0.015 & 0.012 & 0.008 \\
\hline & $\mathrm{T}(\mathrm{c}, \mathrm{p})$ & 0.062 & 0.094 & 0.165 & 0.121 & 0.134 & 0.153 & 0.154 & 0.139 & 0.130 \\
\hline
\end{tabular}




\begin{tabular}{|c|c|c|c|c|c|c|c|c|c|c|}
\hline \multirow{5}{*}{ South-west } & $\mathrm{T}(\mathrm{es}, \mathrm{p})$ & 0.003 & 0.009 & 0.008 & 0.006 & 0.006 & 0.011 & 0.007 & 0.009 & 0.011 \\
\hline & $\mathrm{T}(\mathrm{ei}, \mathrm{p})$ & 0.055 & 0.085 & 0.067 & 0.095 & 0.098 & 0.094 & 0.099 & 0.107 & 0.105 \\
\hline & $\mathrm{T}(\mathrm{is}, \mathrm{p})$ & 0.010 & 0.018 & 0.005 & 0.010 & -0.002 & -0.002 & -0.005 & -0.006 & -0.009 \\
\hline & $\mathrm{T}(\mathrm{gp}, \mathrm{p})$ & 0.011 & 0.015 & 0.013 & 0.016 & 0.004 & 0.013 & 0.011 & 0.012 & 0.003 \\
\hline & $\mathrm{T}(\mathrm{c}, \mathrm{p})$ & 0.080 & 0.127 & 0.094 & 0.126 & 0.106 & 0.117 & 0.113 & 0.121 & 0.110 \\
\hline
\end{tabular}

That is to say, relatively developed areas, such as Jingjin Region and Eastern Coastal Region, are able to narrow the internal provinces or cities' differences in energy structure to reduce carbon inequality. Whereas, energy intensities within less developed regions are still diversified, the differences increased the inequality. As the pace of industrial layout, industrial structure in Northeast Region and Jingjin Region has widened, oppositely, Southern Coastal and Northwest Region narrow that gap, which brings the positive and negative impacts on the regional carbon inequality correspondingly.

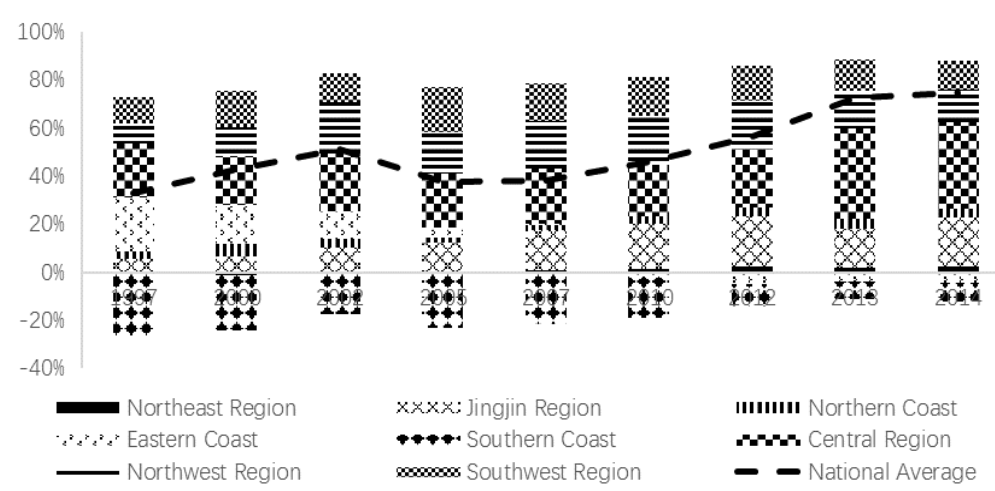

Figure 9. The Theil index of per capita $\mathrm{CO}_{2}$ emission in China from 1997-2014 (Intra-regional)

Table 5. The main influencing factors of per capita $\mathrm{CO}_{2}$ emission in 8 regions from 1997-2014

\begin{tabular}{lc}
\hline Region & Main Influencing Factors \\
\hline Northest & $\mathrm{EI}(+), \mathrm{IS}(+)$ \\
Jingjin & $\mathrm{ES}(+), \mathrm{IS}(+)$ \\
Northern Coast & $\mathrm{EI}(+), \mathrm{PG}(-)$ \\
Eastern Coast & $\mathrm{ES}(-), \mathrm{PG}(+)$ \\
Southern Coast & $\mathrm{EI}(+), \mathrm{IS}(-)$ \\
Central & $\mathrm{EI}(+)$ \\
Northwest & $\mathrm{EI}(+), \mathrm{IS}(-)$ \\
Southwest & $\mathrm{EI}(+)$ \\
\hline
\end{tabular}

Table 6. The main influenced regions of 4 influencing factors

\begin{tabular}{ll}
\hline Influencing Factor & Influenced Regions \\
\hline ES & Jingin, Eastern Coast(-) \\
EI & Northeast, Northern Coast, Southern Coast, Central, Northwest, Southwest(+) \\
IS & Northeast(+), Jingjin(+), Southern Coast(-), Northwest(-) \\
PG & Northern Coast(-) \\
\hline
\end{tabular}

\section{Conclusions and Implications}

We combine the Theil Index and KAYA decomposition technique to analyze the inter-and intra-regional inequity of per capita $\mathrm{CO}_{2}$ in China's from 1997 to 2014, and analyze effects of energy structure, energy intensity, industrial structure, and per capita GDP on inequality. This study finds that:

The regional carbon inequity has an inverse relationship with economic development. In good times, regional inequality of $\mathrm{CO}_{2}$ emissions per capita declines when economic growth rate rises. On the contrary, when economic growth declines, regional carbon inequality rises.

High $\mathrm{CO}_{2}$ emissions per capita is supported by economic prosperity. In 1997-2014, China's per capita $\mathrm{CO}_{2}$ 
emissions have been through three stages, namely moderate stage from 1997 to 2002, high speed stage from 2002 to 2007, and medium stage from 2007 to 2014 respectively. At the same time, regional inequality of per capita $\mathrm{CO}_{2}$ emissions also presents an 'expand-shrink-expand' three-stage feature accordingly. That is to say, when per capita $\mathrm{CO}_{2}$ emissions reach its high point, its regional inequality reaches the low point, and vice versa.

Factors have diverse effects on regions. Per capita GDP factor decreases the inequality, whereas factors of energy efficiency increase the inequality. Meanwhile, more developed areas tend to have higher energy efficiency, and less developed areas are accompanied with lower energy efficiency.

Among the 4 influencing factors, Per capita GDP is the main negative factor, and Energy Structure and Energy Intensity are the main positive factors, and their effects expand over time. That is to say, economic growth is beneficial to reduce regional carbon inequality, and regional differences of energy structure and energy efficiency are the major sources of inequality.

Specifically, in terms of energy structure, the proportions of coal usage in total energy are diversified among regions, leading to an obvious regional energy structure differentiation. In terms of energy intensity, regions cluster into three groups, which are high energy intensity area (Northwest Region), low energy intensity areas (Jingjin Region, Eastern Coastal and Southern Coastal) and medium energy intensity areas (the rest regions). Also, the higher energy intensity areas are less developed areas, and the lower energy intensity areas are more developed areas. That explains the negative relationship between economic development and energy efficiency. Developed areas have abilities to improve the energy using technology and energy structure, thus tend to have improved energy efficiency.

Regions present different carbon inequity characteristics, wherein Energy Efficiency is the main influencing factor. Inequalities of $\mathrm{CO}_{2}$ emissions per capita in more developed regions decline gradually, moreover, Eastern Coast and Southern Coast even appear negative inequality, illustrating internal carbon converge within regions. Besides more developed regions are able to optimize energy structure to reduce the inequality. On the contrary, less developed areas are expanding the inequality, with uneven regional energy efficiency and the gap is widening.

Based on the above conclusions, following policy implications are given.

(1) According to characteristics of regions, to formulate regional emission reduction targets.

Different regions have different factors influencing the levels of $\mathrm{CO}_{2}$ emissions. The Northwest, Central and Northern Coastal have higher $\mathrm{CO}_{2}$ emissions per capita level, these areas also have higher proportions of coal use in the energy structures and energy intensities, but have big differences in industrial structure and per capita output level. The Southwest and Southern Coastal have lower per capita $\mathrm{CO}_{2}$ emission levels, but in the southwest that is due to the relatively low level of per capita output, and that of Southern Coast is due to lower energy intensity.

Therefore, when designing emission reduction targets, different aspects should be focused across regions. For more developed regions, such as the Jing-jin Region, Eastern Coastal and Southern Coastal regions, their output per head level is higher, but have lower energy intensity, and have low proportions to the use of coal in energy structure. Hence, the principle of emission reduction in these regions should take the advantage of economic growth, and further improve the energy efficiency. For less developed areas, such as the Central, Northwest and Southwest regions, they have relatively lower per capita output, but the energy intensity is high, besides the use of coal in the energy structure is higher. Controlling the output per capita to limit carbon emissions in these regions has limited space, and they tend to lower the energy intensity when considering the reduction principle. What's more, in the Northeast and the Northern Coastal areas, the industry structures are relatively high, and the per capita output and the energy efficiency are at the average level, so it is applicable to the principle of transformation of industrial structure.

(2) Reducing regional inequity of per capita $\mathrm{CO}_{2}$ emissions based on regional characteristics.

The carbon inequities of more developed regions, such as Jing-jin region, the Eastern Coastal and Southern Coastal Region, mainly come from their internal energy structure and industrial structure differences. Whereas inequalities of other areas mainly come from the differences in energy intensities. Therefore, to coordinate the regional inequality of $\mathrm{CO}_{2}$ emissions per capita, policy makes should adopt appropriate strategies in order to suit the regional environment. For more developed regions, special funds could be set up to actively promote the sharing of energy use technology, complementary with fiscal and financial incentives to drive cities and enterprises to build clean energy production lines, spread the use of clean energy technology, optimize energy structure, and set rules about the proportion of coal in energy use. At the same time, we can adjust the industrial 
structures in these regions, by eliminating excess capacity or upgrading traditional industries. For other areas, the priorities are to reduce the difference of regional energy intensities, and by introducing clean energy technology to improve the efficiency of energy use.

(3) Overall, increase the energy efficiency, and adjust the industrial structure.

Energy efficiency significantly influence the level of China's per capita $\mathrm{CO}_{2}$ emissions, also the carbon inequality. Upgrading the energy technology and improving the energy efficiency to create low carbon energy structures, can be beneficial for China's $\mathrm{CO}_{2}$ emission levels and the regional carbon inequality. On one hand, local governments and enterprises are encouraged to introduce and develop advanced energy technologies. On the other hand, regional communications of energy using technologies are encouraged. In that way, regional energy use technology sharing mechanism can be established. At the same time, new energy sources can be developed to local conditions, such as the hydroenergy in Central region, wind energy in Northwest Region. When optimizing the energy structure, local industrial structure can also be optimized, and economic growth target and $\mathrm{CO}_{2}$ emissions reduction targets can be connected.

\section{References}

Bourguignon, F. (1979). Decomposable income inequality measures. Econometrica: Journal of the Econometric Society, 901-920. http://dx.doi.org/10.2307/1914138

Cantore, N., \& Padilla, E. (2010). Equality and $\mathrm{CO}_{2}$ emissions distribution in climate change integrated assessment modelling. Energy, 35(1), 298-313. https://doi.org/10.1016/j.energy.2009.09.022

Clarke-Sather, A., Qu, J., Wang, Q., Zeng, J., \& Li, Y. (2011). Carbon inequality at the sub-national scale: A case study of provincial-level inequality in $\mathrm{CO}_{2}$ emissions in China 1997-2007. Energy Policy, 39(9), 5420-5428. https://doi.org/10.1016/j.enpol.2011.05.021

Duro, J. A., \& Padilla, E. (2006). International inequalities in per capita $\mathrm{CO}_{2}$ emissions: A decomposition methodology by Kaya factors. Energy Economics, 28(2), 170-187. https://doi.org/10.1016/j.eneco.2005.12.004

Groot, L. (2010). Carbon Lorenz curves. Resource and Energy Economics, 32(1), 45-64. http://dx.doi.org/10.1016/j.reseneeco.2009.07.001

Heil, M. T., \& Wodon, Q. T. (1997). Inequality in $\mathrm{CO}_{2}$ emissions between poor and rich countries. The Journal of Environment \& Development, 6(4), 426-452. http://dx.doi.org/10.1177/107049659700600404

Heil, M. T., \& Wodon, Q. T. (2000). Future inequality in CO2 emissions and the impact of abatement proposals. $\begin{array}{lllll}\text { Environmental and } & \text { Resource 163-181. }\end{array}$ https://pdfs.semanticscholar.org/7eae/dffebca03d28c8f5cc3ad7687c09fd611114.pdf

Padilla, E., \& Serrano, A. (2006). Inequality in $\mathrm{CO}_{2}$ emissions across countries and its relationship with income inequality: A distributive approach. Energy Policy, 34(14), 1762-1772.

Pan, J. H., \& Zhang, L. F. (2011). A study of areal carbon productivity in China. China Industrial Economics, $5(4), 57$.

Theil, H. (1967). Economics and information theory.

Wang, D., Nie, R., \& Wang, S. Z. (2012). The measurement and decomposition of unequal carbon dioxide emissions in China. Studies in Science of Science, 30(11), 1662-1670.

Yang, J., \& Liu, H. J. (2012). Regional differences in carbon dioxide emissions in China. Quantitative \& Technical Economics, 5, 36-49.

\section{Notes}

Note 1. Carbon Intensity of certain energy changes little with time.

Note 2. Multi-Regional Input Output Table divide China into 8 regions. Due to the availability of data, we follow the same way.

Note 3. Based on our estimation, carbon emission by residential consumption accounts for $3.39 \%$ on average from 1997-2014.

Note 4 . We subtract petroleum refineries and gas works input, but add back usages of cleaned coal and other washed coal. 
Note 5. Industry Sector contributes more than $90 \%$ of the total Carbon Dioxide.

Note 6. The usage of Raw Coal and Coke accounts for about $85 \%$ of total energy use on average.

Note 7. The unit is tce/ten thousand yuan.

Note 8 . The average contribution rate to carbon dioxide emissions of industry sector is $91.41 \%$ in $2002-2007$, $88.54 \%$ in $2008-201,99.62 \%$ in $2013-2014$.

\section{Copyrights}

Copyright for this article is retained by the author(s), with first publication rights granted to the journal.

This is an open-access article distributed under the terms and conditions of the Creative Commons Attribution license (http://creativecommons.org/licenses/by/4.0/). 